\title{
Audição e linguagem em crianças deficientes auditivas implantadas inseridas em ambiente bilíngue: um estudo de casos
}

\author{
Auditory and language abilities in children with cochlear \\ implants who live in bilingual homes: a cases report
}

\author{
Tatiana Mendes de Melo , Elisabete Honda Yamaguti², Adriane Lima Mortari Moret ${ }^{3}$, Maria Cecília Bevilacqua ${ }^{3}$
}

\begin{abstract}
RESUMO
O implante coclear (IC) tem sido indicado para crianças deficientes auditivas de grau severo e/ou profundo que não tem benefício com o aparelho de amplificação sonora individual (AASI), e que apresentem família adequada e motivada para o uso do dispositivo, bem como condições adequadas de reabilitação na cidade de origem. Atualmente, a procura pelo IC também ocorre por pais surdos, fluentes na Língua Brasileira de Sinais (LIBRAS), que recorrem a este tratamento para oferecer outra realidade para seus filhos. O ambiente destas crianças é bilíngue, dado pela LIBRAS dos pais e pela linguagem oral dos familiares próximos, do fonoaudiólogo e da escola. Neste sentido, o presente estudo visou acompanhar quatro crianças deficientes auditivas implantadas, sendo duas crianças filhas de pais deficientes auditivos fluentes na LIBRAS (expostas a ambiente bilíngue) e duas crianças filhas de pais sem alterações auditivas (expostas a ambiente oral). Para tanto, as habilidades de audição e de aquisição da linguagem oral foram comparadas nas quatro crianças implantadas. Foi possível observar que as quatro crianças apresentaram habilidades auditivas e de linguagem semelhantes ao longo do primeiro ano de uso do IC. Contudo, a partir disto, as crianças inseridas em ambiente bilíngue apresentaram melhor desempenho auditivo e linguístico, comparado ao desenvolvimento das outras crianças. As crianças inseridas em ambiente bilíngue podem se beneficiar do IC, desenvolvendo habilidades auditivas e de linguagem similares às das crianças inseridas em ambiente oral. Ressalta-se que os benefícios do dispositivo são obtidos a partir de aspectos multifatoriais, e estudos mais aprofundados são necessários.
\end{abstract}

Descritores: Perda auditiva; Implante coclear; Linguagem; Multilinguismo; Reabilitação de deficientes auditivos

\section{INTRODUÇÃO}

O Implante Coclear (IC) representa o mais importante avanço no tratamento de deficientes auditivos de grau severo e/ou profundo bilateral que não apresentam aproveitamento com o aparelho de amplificação sonora individual (AASI), possibilitando melhores resultados na trajetória de desenvolvimento auditivo, linguístico, social e acadêmico.

No Brasil, todo o processo para a realização e acompanhamento do IC é garantido pelo Sistema Único de Saúde (SUS)

Trabalho realizado no Centro de Pesquisas Audiológicas, Hospital de Reabilitação de Anomalias Craniofaciais, Universidade de São Paulo - USP

-Bauru (SP), Brasil.

Conflito de interesses: Não

(1) Núcleo do Ouvido Biônico, Hospital Samaritano - São Paulo (SP), Brasil.

(2) Centro de Pesquisas Audiológicas, Hospital de Reabilitação de Anomalias

Craniofaciais, Universidade de São Paulo - USP -Bauru (SP), Brasil.

(3) Departamento de Fonoaudiologia, Faculdade de Odontologia de Bauru, Universidade de São Paulo - USP -Bauru (SP), Brasil.

Endereço para correspondência: Maria Cecília Bevilacqua. R. Silvio Marchione, 3/20, Vl. Universitária, Bauru (SP), Brasil, CEP: 17012-900. E-mail: cpa@ centrinho.usp.br

Recebido em: 20/10/2011; Aceito em: 12/4/2012 desde 1993, a partir dos critérios de indicação e contraindicação do dispositivo para crianças e adultos, estabelecidos pelo Ministério da Saúde.

De acordo com esta Portaria, nascrianças com idade inferior a 18 anos de idade, com deficiência auditiva neurossensorial bilateral de grau severo e/ou profundo, de caráter pré ou pós-lingual, o IC deverá ser indicado a partir dos seguintes critérios: experiência com AASI, durante pelo menos três meses; incapacidade de reconhecimento de palavras em conjunto fechado; família adequada e motivada para o uso do dispositivo; condições adequadas de reabilitação na cidade de origem.

Tais critérios também podem variar de acordo com o programa de cada instituição credenciada, contudo aspectos como diagnóstico da deficiência auditiva, idade do paciente, benefício com o uso da amplificação, existência de programas educacionais na cidade de origem e motivação/expectativa da família sempre são considerados no momento da indicação do dispositivo.

Na proposta do Bilinguismo, a linguagem oral é desenvolvida como segunda língua do deficiente auditivo, enquanto que a língua de sinais é adquirida como a principal forma de comunicação ${ }^{(1)}$. 
No âmbito internacional há uma crescente procura do IC pela comunidade bilíngue como tratamento para o deficiente auditivo $^{(2-4)}$, a partir do reconhecimento pela National Association of the Deaf sobre a importância do IC na qualidade de vida desta população ${ }^{(5,6)}$. No contexto nacional também ocorre abusca pelo dispositivo por pais deficientes auditivos, fluentes na Língua Brasileira de Sinais (LIBRAS), que recorrem a este tratamento para oferecer uma opção de reabilitação para seus filhos. O ambiente destas crianças é bilíngue, dado pela LIBRAS dos pais e pela linguagem oral dos familiares próximos, da escola, e da terapia fonoaudiológica.

Neste sentido, torna-se necessário conhecer o perfil de desenvolvimento das habilidades de linguagem e auditivas de crianças deficientes auditivas implantadas no país e que são expostas aambiente bilíngue.

\section{APRESENTAÇÃO DO CASO CLÍNICO}

O presente estudo visou acompanhar, longitudinalmente, crianças deficientes auditivas implantadas, filhas de pais deficientes auditivos fluentes em LIBRAS. Ambas as duplas de crianças são atendidas no Centro de Pesquisas Audiológicas do Hospital de Reabilitação de Anomalias Craniofaciais da Universidade de São Paulo (CPA/HRAC-USP) e receberam a indicação cirúrgica do dispositivo por se enquadrarem nos critérios de indicação de IC deste centro. Cabe ressaltar que a indicação foi realizada, considerando a solicitação dos pais e a inserção das crianças em um ambiente ouvinte por parte dos familiares ouvintes.

Para tanto, participaram deste estudo quatro crianças, sendo duas crianças inseridas em ambiente bilíngue (denominadas no estudo como criança A e B) e duas crianças inseridas em ambiente ouvinte(denominadas no estudo como criança A1 e B1), ou seja, crianças com pais sem alterações auditivas.

$\mathrm{Na}$ etapa pré-cirúrgica as crianças inscritas neste serviço de IC são submetidas à avaliação otorrinolaringológica, avaliação fonoaudiológica, avaliação psicológica, avaliação social, exames de imagem, e demais avaliações complementares, dependendo da necessidade de cada paciente.

Nesta etapa todas as crianças são avaliadas quanto ao estilo cognitivo, para observação e registro de alguns comportamentos que fazem parte do desenvolvimento infantil, e outros que, quando presentes, podem ser indicadores de patologias ou dificuldades específicas da criança, impeditivos de um desenvolvimento pleno. Deste modo, quanto maior o escore obtido, mais compatível está o desenvolvimento global da criança avaliada, comparado aos marcos do desenvolvimento na infância. As famílias também são avaliadas de acordo com critérios de referência quanto ao grau de permeabilidade ao processo terapêutico, e, quanto maior o escore obtido, maior o envolvimento da família na reabilitação auditiva da criança. Ambas as avaliações são realizadas com protocolo interno do CPA/HRAC-USP.

As informações referentes ao perfil audiológico das crianças estudadas, antes da cirurgia do IC estão disponíveis na Tabela 1. Por sua vez, os dados referentes ao estilo cognitivo da criança, à permeabilidade da família no processo terapêutico e o nível socioeconômico podem ser visualizados na Tabela 2. A classificação socioeconômica utilizada pelo serviço de IC divide-se em: alta (A), média superior (MS), média (ME),

Tabela 1. Perfil audiológico das crianças estudadas, antes da cirurgia do implante coclear

\begin{tabular}{|c|c|c|c|c|c|c|c|c|c|c|c|}
\hline \multirow[t]{2}{*}{ Criança } & \multirow{2}{*}{$\begin{array}{l}\text { Etiologia da deficiência } \\
\text { auditiva }\end{array}$} & \multicolumn{4}{|c|}{$\begin{array}{l}\text { Limiares auditivos pré-IC } \\
\text { (sem AASI) }\end{array}$} & \multicolumn{4}{|c|}{$\begin{array}{l}\text { Limiares auditivos pré-IC } \\
\text { (com AASI) }\end{array}$} & \multirow{2}{*}{$\begin{array}{c}\text { Uso efetivo } \\
\text { AASI } \\
\text { pré-IC }\end{array}$} & \multirow[t]{2}{*}{ Modelo do AASI } \\
\hline & & $0,5 \mathrm{kHz}$ & $1 \mathrm{kHz}$ & $2 \mathrm{kHz}$ & $4 \mathrm{kHz}$ & $0,5 \mathrm{kHz}$ & $1 \mathrm{kHz}$ & $2 \mathrm{kHz}$ & $4 \mathrm{kHz}$ & & \\
\hline A & Genética & $\downarrow 100 \mathrm{~dB}$ & $\downarrow 100 \mathrm{~dB}$ & $\downarrow 100 \mathrm{~dB}$ & $\downarrow 100 \mathrm{~dB}$ & $65 \mathrm{~dB}$ & $75 \mathrm{~dB}$ & $80 \mathrm{~dB}$ & $80 \mathrm{~dB}$ & Sim & 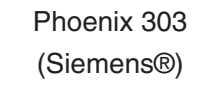 \\
\hline A1 & Rubéola congênita & $90 \mathrm{~dB}$ & $100 \mathrm{~dB}$ & $\downarrow 100 \mathrm{~dB}$ & $\downarrow 100 \mathrm{~dB}$ & $80 \mathrm{~dB}$ & $95 \mathrm{~dB}$ & $\downarrow 100 \mathrm{~dB}$ & $\downarrow 100 \mathrm{~dB}$ & Sim & $\begin{array}{l}\text { Phoenix } 303 \\
\text { (Siemens } ®)\end{array}$ \\
\hline B & $\begin{array}{l}\text { Sindrome de } \\
\text { Waanderburg }\end{array}$ & $\downarrow 100 \mathrm{~dB}$ & $\downarrow 100 \mathrm{~dB}$ & $\downarrow 100 \mathrm{~dB}$ & $\downarrow 100 \mathrm{~dB}$ & $75 \mathrm{~dB}$ & $85 \mathrm{~dB}$ & $\downarrow 100 \mathrm{~dB}$ & $\downarrow 100 \mathrm{~dB}$ & Sim & $\begin{array}{l}\text { Phoenix } 303 \\
\text { (Siemens } ®)\end{array}$ \\
\hline B1 & Rubéola congênita & $75 \mathrm{~dB}$ & $85 \mathrm{~dB}$ & $80 \mathrm{~dB}$ & $95 \mathrm{~dB}$ & $65 \mathrm{~dB}$ & $50 \mathrm{~dB}$ & $60 \mathrm{~dB}$ & $70 \mathrm{~dB}$ & Sim & 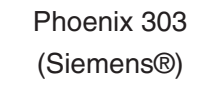 \\
\hline
\end{tabular}

Nota: 0,5; 1 k;2k e 4khz - campo livre

Legenda: $\mathrm{IC}=$ implante coclear; $\downarrow 100 \mathrm{~dB}=$ limiar auditivo ausente em $100 \mathrm{~dB}$ (máximo do equipamento); Crianças $\mathrm{A}$ e $\mathrm{B}=$ inseridas em ambiente bilíngue; Crianças $\mathrm{A} 1$ e $\mathrm{B} 1$ = inseridas em ambiente oral

Tabela 2. Dados referentes ao estilo cognitivo da criança, a permeabilidade da família no processo terapêutico e nível sócio-econômico da família das crianças estudadas

\begin{tabular}{|c|c|c|c|c|}
\hline Criança & $\begin{array}{l}\text { Estilo cognitivo da criança } \\
\qquad(\%)\end{array}$ & $\begin{array}{l}\text { Permeabilidade da família } \\
\qquad(\%)\end{array}$ & $\begin{array}{l}\text { Nível sócio-econômico da } \\
\text { família }\end{array}$ & $\begin{array}{c}\text { Grau de escolaridade da } \\
\text { mãe/pai }\end{array}$ \\
\hline$A$ & 71,20 & 77,50 & E-BS & Ensino médio completo \\
\hline A1 & 80,00 & 95,00 & E-BS & Ensino médio completo \\
\hline B & 70,84 & 85,00 & D-MI & Ensino médio completo \\
\hline B1 & 97,92 & 93,00 & E-BS & Ensino médio completo \\
\hline
\end{tabular}

Legenda: $\mathrm{E}-\mathrm{BS}$ = Classe $\mathrm{E}$ - baixa superior; $\mathrm{D}-\mathrm{MI}=$ Classe $\mathrm{D}$ - média inferior; Crianças $\mathrm{A}$ e $\mathrm{B}=$ inseridas em ambiente bilíngue; Crianças $\mathrm{A} 1$ e $\mathrm{B} 1$ = inseridas em ambiente oral 
média inferior (MI), baixa superior (BS) e baixa (BA), de acordo com a renda familiar (em salários mínimos), número de membros residentes da família, maior nível educacional da família, tipo e condição habitacional e o maior nível ocupacional.

Ambas as duplas apresentam deficiência auditiva neurossensorial de grau profundo, de origem pré-lingual, usuárias de IC modelo Nucleus 24K (Cochlear Corporation®). Em todos os casos houve inserção total dos eletrodos. Para todas as crianças o processador de fala foi programado com a estratégia de codificação de fala ACE (Advanced Combination Encoders) e máxima espectral igual a 12. Ambas as duplas de crianças eram usuárias de AASI anteriormente à cirurgia de IC e estão inseridas em um programa de reabilitação auditiva com abordagem aurioral.

As habilidades de audição e de linguagem oral das duas crianças inseridas em ambiente bilíngue foram comparadas com as habilidades das duas crianças inseridas em ambiente ouvinte, pareadas quanto à idade cronológica, tempo de privação sensorial e tempo de uso do IC.

Todos os responsáveis pelas crianças do presente estudo assinaram o Termo de Consentimento Livre e Esclarecido, consentindo assim com a realização e divulgação de seus resultados.

Foi realizado o levantamento de dados contidos nos prontuários dos pacientes, referentes ao desempenho de percepção auditiva da fala e de aquisição de linguagem, de acordo com as categorias de audiçãa ${ }^{(7)}$ e de linguagem ${ }^{(8)}$, conforme apresentado no Anexo 1. Informações referentes às variáveis: uso do IC, terapia fonoaudiológica e escola também foram coletadas para o presente estudo.

Os procedimentos para atribuição das categorias de audição foram: avaliação clínica de comportamento auditivo; Teste de Avaliação da Capacidade Auditiva Mínima (TACAM) ${ }^{(9)}$; Infant Toddler: Meaningful Auditory Integration Scale (IT-MAIS) ${ }^{(10)}$;
Procedimento para a Avaliação de Crianças Deficientes Auditivas Profundas ${ }^{(11)}$; e Lista de palavras como procedimento de avaliação da percepção dos sons da fala ${ }^{(12)}$.

Os procedimentos de avaliação para a atribuição de categorias de linguagem foram: avaliação da atitude de comunicação oral em situação de interação lúdica e em atividades direcionadas com a avaliadora e com o acompanhante da criança; questionário Meaningful Use of Speech Scales(MUSS) ${ }^{(13)}$.

As habilidades de audição e de linguagem oral das crianças foram avaliadas ao longo do processo de acompanhamento pós-operatório, conforme observado na Tabela 3. As informações referentes às variáveis que podem influenciar o desenvolvimento das habilidades estudadas podem ser visualizadas na Tabela 4.

Ambas as duplas (A e A1; B e B1) apresentaram habilidades auditivas e de linguagem semelhantes, ao longo do primeiro ano de uso do IC. Contudo, a partir disto, as crianças inseridas em ambiente bilíngue apresentaram melhor desempenho auditivo e linguístico, comparado ao desenvolvimento das outras crianças.

Do ponto de vista auditivo, as crianças "A" e "B" (ambiente bilíngue) atingiram a habilidade de reconhecimento auditivo em conjunto aberto (capacidade de ouvir palavras fora do contexto e identificar a palavra exclusivamente por meio da audição) após dois e três anos de IC, respectivamente. Por sua vez, a criança inseridaem ambiente oral (criança "A1") atingiu esta habilidade após quatro anos de IC e a criança "B1" não atingiu esta habilidade até a última avaliação proposta por este estudo (cinco anos de uso do dispositivo).

Em termos de desenvolvimento linguístico, as crianças "A" e "B" (ambiente bilíngue), após três anos de uso do IC eram capazes de construir frases de mais de cinco palavras, usando elementos conectivos, conjugando verbos, utilizando plurais, ou seja, apresentando fluência na linguagem oral (categoria

Tabela 3. Evolução das habilidades auditivas e de linguagem das crianças estudadas

\begin{tabular}{|c|c|c|c|c|c|c|c|c|c|c|c|c|c|c|c|c|c|c|c|c|c|}
\hline \multirow[t]{2}{*}{ Criança } & \multirow[t]{2}{*}{$\begin{array}{l}\text { Idade na } \\
\text { cirurgia }\end{array}$} & \multicolumn{2}{|c|}{ Pré IC } & \multicolumn{2}{|c|}{$\begin{array}{c}\text { Ativação } \\
\text { IC }\end{array}$} & \multicolumn{2}{|c|}{$\begin{array}{c}3 \text { meses } \\
\text { de IC }\end{array}$} & \multicolumn{2}{|c|}{$\begin{array}{c}6 \text { meses } \\
\text { de IC }\end{array}$} & \multicolumn{2}{|c|}{$\begin{array}{l}1 \text { ano } \\
\text { de IC }\end{array}$} & \multicolumn{2}{|c|}{$\begin{array}{c}1 \text { ano e } 6 \\
\text { meses de } \\
\text { IC }\end{array}$} & \multicolumn{2}{|c|}{$\begin{array}{c}2 \text { anos } \\
\text { de IC }\end{array}$} & \multicolumn{2}{|c|}{$\begin{array}{c}3 \text { anos } \\
\text { de IC }\end{array}$} & \multicolumn{2}{|c|}{$\begin{array}{c}4 \text { anos } \\
\text { de IC }\end{array}$} & \multicolumn{2}{|c|}{$\begin{array}{c}5 \text { anos } \\
\text { de IC }\end{array}$} \\
\hline & & $\mathrm{CA}$ & $\mathrm{CL}$ & $\mathrm{CA}$ & $\mathrm{CL}$ & $\mathrm{CA}$ & $\mathrm{CL}$ & $\mathrm{CA}$ & $\mathrm{CL}$ & $\mathrm{CA}$ & $\mathrm{CL}$ & $\mathrm{CA}$ & $\mathrm{CL}$ & $\mathrm{CA}$ & $\mathrm{CL}$ & $\mathrm{CA}$ & $\mathrm{CL}$ & $\mathrm{CA}$ & $\mathrm{CL}$ & $\mathrm{CA}$ & $\mathrm{CL}$ \\
\hline A & 22 meses & 1 & 1 & 1 & 1 & 1 & 1 & 2 & 2 & 2 & 2 & 4 & 3 & 6 & 3 & 6 & 5 & 6 & 5 & 6 & 5 \\
\hline A1 & 22 meses & 1 & 1 & 1 & 1 & 1 & 1 & 2 & 2 & 2 & 2 & 3 & 3 & 3 & 3 & 4 & 4 & 6 & 4 & 6 & 4 \\
\hline B & 12 meses & 0 & 0 & 1 & 1 & 1 & 1 & 2 & 2 & 3 & 3 & 4 & 3 & 5 & 4 & 6 & 5 & 6 & 5 & 6 & 5 \\
\hline B1 & 12 meses & 1 & 1 & 1 & 1 & 2 & 2 & 2 & 2 & 2 & 2 & 2 & 2 & 2 & 2 & 4 & 3 & 4 & 3 & 5 & 4 \\
\hline
\end{tabular}

Legenda: IC =implante coclear; $C A$ =categoria de audição; $C L=$ categoria de linguagem; Crianças $A$ e $B=$ inseridas em ambiente bilíngue; Crianças $A 1$ e $B 1$ = inseridas em ambiente oral

Tabela 4. Informações sobre as variáveis que podem influenciar o desenvolvimento das habilidades estudadas

\begin{tabular}{|c|c|c|c|c|c|c|}
\hline Criança & Uso efetivo do IC & $\begin{array}{l}\text { Uso do AASI } \\
\text { contralateral }\end{array}$ & $\begin{array}{c}\text { Abordagem terapia } \\
\text { fonoaudiológica }\end{array}$ & $\begin{array}{l}\text { Frequência semanal da } \\
\text { terapia fonoaudiológica }\end{array}$ & $\begin{array}{l}\text { Duração da sessão de } \\
\text { terapia fonoaudiológica }\end{array}$ & Escola regular \\
\hline$A$ & Sim & Não & Aurioral & 3 vezes & 50 minutos & Sim \\
\hline A1 & Sim & Não & Aurioral & 2 vezes & 60 minutos & Sim \\
\hline B & Sim & Não & Aurioral & 2 vezes & 50 minutos & Sim \\
\hline B1 & Sim & Não & Aurioral & 2 vezes & 45 minutos & Sim \\
\hline
\end{tabular}

Legenda: $\mathrm{IC}$ = implante coclear; $\mathrm{AASI}=$ aparelho de amplificação sonora individual; Crianças A e B = inseridas em ambiente bilíngue; Crianças A1 e B1 = inseridas em ambiente oral 
de linguagem cinco). As crianças inseridas em ambiente oral ("A1" e "B1"), após cinco anos de uso do IC, ainda não atingiram esta habilidade.

\section{DISCUSSÃO}

Em função da crescente procura do IC por pais deficientes auditivos, fluentes na Língua Brasileira de Sinais (LIBRAS), torna-se importante relacionar evidências científicas a respeito do desenvolvimento das habilidades auditivas e de linguagem destas crianças ${ }^{(3)}$, com o intuito de auxiliar na indicação do dispositivo nos diversos Programas de IC do país.

De acordo com os resultados do presente estudo, as crianças inseridas em ambiente bilíngue podem se beneficiar do IC, desenvolvendo habilidades auditivas e de linguagem oral similares ou melhores que as crianças inseridas em ambiente oral $^{(3,14)}$. O desenvolvimento destas habilidades pelas crianças implantadas está relacionado com as características individuais de cada paciente, como também com a reabilitação auditiva e a permeabilidade da família neste processo $^{(15)}$. Desta maneira, reforça-se a importância da participação dos familiares próximos a estas crianças inseridas em ambiente bilíngue, que proporcionaram o ambiente rico em experiências auditivas para a aquisição destas habilidades pelas crianças.

Todas as crianças estudadas faziam uso efetivo do IC, mas não utilizavam o AASI contralateral (Tabela 4). As mesmas estavam inseridas em terapia fonoaudiológica com abordagem aurioral, frequentando efetivamente a terapia e a escola regular. Desta forma, é possível observar que a homogeneidade das crianças estudadas em relação a estes aspectos avaliados parece não ter influenciado no melhor desempenho auditivo e linguístico das crianças inseridas em ambiente bilíngue.

O mesmo é possível afirmar em relação aoutros aspectos avaliados, como a permeabilidade da família no processo terapêutico, o estilo cognitivo da criança, o nível socioeconômico da família e o grau de escolaridade dos pais das crianças avaliadas, isto é, tais variáveis foram bastante semelhantes nas duplas de crianças estudadas e parecem não explicar o desenvolvimento auditivo e linguístico superior das crianças inseridas no ambiente bilíngue ao longo do uso do IC (Tabela 2).

Os critérios de indicação do IC nos serviços habilitados pelo Ministério da Saúde não inviabiliza a implantação de crianças inseridas em ambiente bilíngue, porém ainda são muitas as indagações sobre os resultados desse dispositivo nestas crianças ao longo do tempo. Uma das mais relevantes diz respeito à influência da língua de sinais na linguagem oral. Pesquisa em âmbito internacional aponta que a partir da aquisição da linguagem oral por essas crianças, não há interferência da língua de sinais no sentido de redução da produção oral e que há uma redução progressiva do uso de sinais ao passo que a linguagem oral começa a ser desenvolvida ${ }^{(4)}$. Corroborando esta pesquisa, as crianças do presente estudo não sofreram nenhuma interferência negativa, do ponto de vista auditivo e linguístico, em função da inserção das mesmas no ambiente bilíngue, uma vez que tais habilidades foram desenvolvidas de forma semelhante aos seus pares inseridos exclusivamente em ambiente oral.

Os pesquisadores ressaltam ainda que, o uso da língua de sinais ou da linguagem oral durante a interação destas crianças depende diretamente de seu interlocutor e do contexto comunicativo. Isto vem ao encontro dos relatos dos familiares das crianças estudadas, que referem que as crianças implantadas muitas vezes incorporavam o papel de intérprete entre o familiar que utilizava LIBRAS e as demais pessoas, ou seja, a criança adapta sua forma de comunicação, de acordo com o interlocutor.

A partir dos resultados expostos, associado à literatura científica, é possível inferir que, se a criança implantada estiver em um ambiente educacional adequado que ofereça a linguagem oral, o conhecimento da língua de sinais não afeta negativamente a evolução das habilidades auditivas e de linguagem. Neste sentido, é importante respeitar a escolha de pais fluentes em LIBRAS em optar pelo IC para o desenvolvimento global da criança deficiente auditiva.

\section{COMENTÁRIOS FINAIS}

Ao longo dos anos, os serviços de IC do país irão abranger cada vez mais a demanda de crianças deficientes auditivas inseridas em ambiente bilíngue. Os profissionais envolvidos nas diferentes etapas da indicação do IC devem estar preparados para o atendimento destas famílias, como também para a avaliação pré-cirúrgica destes casos, de forma minuciosa, uma vez que a indicação do dispositivo estará intimamente relacionada com a inserção desta criança em ambiente oral proporcionado por familiares, fonoaudiólogo e contexto escolar. 


\begin{abstract}
The cochlear implant (CI) has been indicated for children with severe and/or profound bilateral hearing loss who do not benefit from hearing aids and have adequate and motivated family for the use of the device, as well as adequate rehabilitation conditions at their hometowns. Currently, the demand for CI also occurs by deaf parents, fluent in Brazilian Sign Language (LIBRAS), who resort to this treatment to offer their children another reality. The environment of these children is bilingual, with exposition to LIBRAS through their parents and to oral language through close relatives, audiologist/speech-language pathologist, and the school. In this sense, the present study aimed to follow-up four implanted deaf children -two with deaf parents fluent in LIBRAS (exposed to a bilingual environment), and two with hearing parents (exposed to oral environment). For this purpose, abilities of hearing and oral language development were compared in these children with CI. It was observed that all four children in this study presented similar language development and auditory skills throughout the first year of CI use. However, after this period, children inserted into a bilingual environment had better auditory and linguistic performance when compared to the other children. Children in bilingual environments can benefit from the CI, developing auditory skills and oral language similarly to children inserted into an oral environment. It is emphasized that the benefits of the device is dependent on several factors, and further studies are needed.
\end{abstract}

Keywords: Hearing loss; Cochlear implantation; Language; Multilingualism; Rehabilitation of hearing impaired

\title{
REFERÊNCIAS
}

1. Brasil. Lei $\mathrm{n}^{\circ} 10.436$, de 24 de abril de 2002. Dispõe sobre a Língua Brasileira de Sinais - LIBRAS. Diário Oficial da União. 25 abr 2002; Seção 1:59.

2. Steinberg A, Brainsky A, Bain L, Montoya L, Indenbaum M, Potsic W. Parental values in the decision about cochlear implantation. Int J Pediatr Otorhinolaryngol. 2000;55(2):99-107.

3. Cassandro E, Niscatri M, Chiarella G, Genovese E, Gallo LV, Catalano M. Development of communication and speech skills after cochlear implant in a sign language child. Acta Otorhinolary Ital. 2003;23(2):8893.

4. Jiménez MS, Pino MJ, Herruzo J. A comparative study of speech development between deaf children with cochlear implants who have been educated with spoken or spoken sign language. Int J Pediatr Otorhinolaryngol. 2009;73(1):109-14.

5. Christiansen JB, Leigh IW. Children with cochlear implants: changing parent and deaf community perspectives. Arch Otolaryngol Head Neck Surg. 2004;130(5):673-7.

6. Pray JL, Jordan IK. The deaf community and culture at a crossroads: issues and challenges. J Soc Work Disabil Rehabil. 2010;9(2):168-93.

7. Geers AE. Techniques for assessing auditory speech perception and lipreading enhancement in Young deaf children. The Volta Review. 1994;96(5):85-96.

8. Bevilacqua MC, Delgado EM, Moret AL. Estudos de casos clínicos de crianças do Centro Educacional do Deficiente Auditivo (CEDAU), do Hospital de Pesquisa e Reabilitação de Lesões Lábio-Palatais - USP. In: XI Encontro Internacional de Audiologia;1996, 30 de março a 02 de abril. Anais: Bauru, Brasil. p. 187.
9. Orlandi AC, Bevilacqua MC. Deficiência auditiva profunda nos primeiros anos de vida: procedimento para a avaliação da percepção da fala. Pró-Fono. 1998;10(2):87-91.

10. Castiquini EA, Bevilacqua MC. Escala de integração auditiva significativa: procedimento adaptado para a avaliação da percepção da fala. Rev Soc Bras Fonoaudiol.2000;4(6):51-60.

11. Bevilacqua MC, Tech EA. Elaboração de um procedimento de avaliação de percepção de fala em crianças deficientes auditivas profundas a partir de cinco anos de idade. In: Mrchesan IQ, Zorzi JL, Gomes IC (ed.). Tópicos em fonoaudiologia. São Paulo: Lovise; 1996. p. 411-33.

12. Delgado EM, Bevilacqua MC. Lista de palavras como procedimento de avaliação da percepção dos sons da fala para crianças deficientes auditivas. Pró-fono. 1999;11(1):59-64.

13. Nascimento LT. Uma proposta de avaliação da linguagem oral. [monografia]. Bauru: Universidade de São Paulo - Hospital de Pesquisa e Reabilitação de Lesões Lábio-Palatais;1997.

14. Thomas E, El-Kashlan H, Zwolan TA. Children with cochlear implants who live in monolingual and bilingual homes. OtolNeurotol. 2008;29(2):230-4.

15. Moret $\mathrm{AL} \mathrm{A}$, Bevilacqua $\mathrm{MC}$, Costa $\mathrm{OA}$. Implante coclear: audição e linguagem em crianças deficientes auditivas pré-linguais. Pró-fono. 2007;19(3):295-304. 
Anexo 1. Categorias de audição e de linguagem

\begin{tabular}{|c|c|c|}
\hline Categoria & Categoria de audição(7) & Categoria de linguagem ${ }^{(8)}$ \\
\hline 0 & $\begin{array}{l}\text { Não detecta a fala } \\
\text { Esta criança não detecta a fala em situações de conversação } \\
\text { normal (limiar de detecção de fala >65 dB). }\end{array}$ & --- \\
\hline 1 & $\begin{array}{l}\text { Detecção } \\
\text { Esta criança detecta a presença do sinal de fala. }\end{array}$ & $\begin{array}{l}\text { Esta criança não fala e pode apresentar vocalizações } \\
\text { indiferenciadas. }\end{array}$ \\
\hline 2 & $\begin{array}{l}\text { Padrão de percepção } \\
\text { Esta criança diferencia palavras pelos traços suprasegmentares } \\
\text { (duração, tonicidade, etc.). Ex: dog X airplane, baby X } \\
\text { birthdaycake (mão X sapato; casa X menino). }\end{array}$ & Esta criança fala apenas palavras isoladas. \\
\hline 3 & $\begin{array}{l}\text { Iniciando a identificação de palavras } \\
\text { Esta criança diferencia palavras em conjunto fechado com base } \\
\text { na informação fonética. Este padrão pode ser demonstrado com } \\
\text { palavras que são idênticas na duração, mas contém diferenças } \\
\text { espectrais múltiplas. Ex: thoothbrush X hot dog, airplane X } \\
\text { lunchbox (geladeira X bicicleta, gato X casa) }\end{array}$ & Esta criança constroi frases de dois ou três palavras. \\
\hline 4 & $\begin{array}{l}\text { Identificação de palavras por meio do reconhecimento da vogal } \\
\text { Esta criança diferencia palavras em conjunto fechado que } \\
\text { diferem primordialmente no som da vogal. Ex: bird, boat, bike, } \\
\text { bat (pé, pó, pá; mão, meu, mim). }\end{array}$ & $\begin{array}{l}\text { Esta criança constroi frases de quatro ou cinco palavras, e } \\
\text { inicia o uso de elementos conectivos (pronomes, artigos, } \\
\text { preposições). }\end{array}$ \\
\hline 5 & $\begin{array}{l}\text { Identificação de palavras por meio do reconhecimento da } \\
\text { consoante } \\
\text { Esta criança diferencia palavras em conjunto fechado que tem } \\
\text { o mesmo som da vogal, mas contém diferentes consoantes. Ex: } \\
\text { hair, pear, chair, stair (mão, pão, tão, cão, chão). }\end{array}$ & $\begin{array}{l}\text { Esta criança constrói frases de mais de cinco palavras, } \\
\text { usando elementos conectivos, conjugando verbos, usando } \\
\text { plurais, etc. Éuma criança fluente na linguagem oral. }\end{array}$ \\
\hline 6 & $\begin{array}{l}\text { Reconhecimento de palavras em conjunto aberto } \\
\text { Esta criança é capaz de ouvir palavras fora do contexto e } \\
\text { extrair bastante informação fonêmica, e reconhecer a palavra } \\
\text { exclusivamente por meio da audição. }\end{array}$ & --- \\
\hline
\end{tabular}

